\title{
磁性金属-有机骨架复合材料的构建及在环境中的 应用
}

\author{
霍江波 ${ }^{1,2}$, 崔浩杰 ${ }^{1}$, 付明来 ${ }^{1 *}$, 苑宝玲 ${ }^{*}$ \\ 1. 中国科学院城市环境研究所, 中国科学院城市污染物转化重点实验室, 厦门 361021 \\ 2. 中国科学院大学, 北京 100049 \\ 3. 华侨大学土木学院, 厦门 361020 \\ *通讯作者, E-mail: mlfu@iue.ac.cn; blyuan@hqu.edu.cn
}

收稿日期: 2016-11-16; 接受日期: 2017-01-02; 网络版发表日期: 2017-04-17

国家自然科学基金(编号: 51478449)资助项目

\begin{abstract}
摘要磁性金属-有机骨架(MMOFs)复合材料是一类新颖的功能材料; MMOFs 在磁性功能粒子的基础上 结合了金属有机骨架材料的孔径均一、比表面积高、化学和热稳定性良好、吸附亲和力强等性能, 在催化 和环境等领域中显示出潜在的应用前景. 本文简要概述了近年来制备的MMOFs复合材料, 包括磁性微球核 壳结构、磁性纳米颗粒镶嵌结构、磁性颗粒无规则互混结构、磁性石墨烯基复合结构; 并从环境污染物的 吸附、富集和催化降解角度, 重点总结了MMOFs 在环境分析和环境修复中的应用。
\end{abstract}

关键词磁性, 金属有机骨架, 复合材料, 环境分析, 环境修复

\section{1 引言}

金属有机-骨架化合物, 又称金属有机配位聚合物 (metal organic frameworks, MOFs), 是一类由金属离子 或金属离子簇通过配位键与有机桥联配体结合形成 的具有无限拓扑结构的网状聚合物 ${ }^{[1]}$. 与传统的多孔 沸石材料相比, MOFs 具有合成简单、种类繁多、孔 径易调、比表面积大 ${ }^{[2]}$ 、化学稳定性好 ${ }^{[3]}$ 、易改性等 优点 ${ }^{[4]}$. MOFs 已广泛应用于气体储存 ${ }^{[5]}$ 、传感 ${ }^{[6]}$ 、生 物医学 ${ }^{[7]}$ 、催化 ${ }^{[8,9]}$ 、环境等领域 ${ }^{[10]}$. 然而, 由于多数 的MOFs 具有难溶于水或有机溶剂、粒径较小、机械 强度低、对水敏感等缺点, 限制了其在某些领域中的 应用 ${ }^{[1]}$. 因此, 功能化MOFs 的研究与开发成为一种新
的需求.

近年来, 报道较多的功能化 $\mathrm{MOFs}$ 主要分为两类: 官能团修饰的 $\mathrm{MOFs}^{[12214]}$ 和功能材料复合的 $\mathrm{MOFs}^{[15]}$. 前者主要是在MOFs 上嫁接活性功能基团 ${ }^{[16]}$, 后者是 将具有特殊功能的材料与MOFs复合. 目前, 许多研究 人员将 $-\mathrm{COOH}^{[17]} 、-\mathrm{NH}_{2}{ }^{[18]} 、-\mathrm{SH}^{[19]} 、-\mathrm{SO}_{3} \mathrm{H}^{[20]}$ 等基团 成功地修饰在 MOFs 上, 并应用于水处理领域. 相比 MOFs, 官能团修饰的MOFs 具有了更高的选择性. 此 外, 研究者还开发了与金属纳米粒子 ${ }^{[21,22] 、}$ 石墨烯 ${ }^{[23]}$ 、 碳纳米管 ${ }^{[24,25]}$ 、磁性纳米粒子 ${ }^{[26]}$ 等结合的 MOFs复合 材料, 这类材料显示出了更优异的性能和潜在的应用 价值. 其中, 磁性MOFs 作为最新开发的一种复合材料, 因具有独特的定位和分离特点而受到了广泛的关注.

引用格式： 霍江波,崔浩杰, 付明来,苑宝玲. 磁性金属-有机骨架复合材料的构建及在环境中的应用. 中国科学: 化学, 2017,47: 830-843 Huo J, Cui H, Fu M, Yuan B. The fabrication of magnetic metal-organic frameworks composites and their application in environment. Sci Sin Chim, 2017, 47: 830-843, doi: 10.1360/N032016-00199 
磁性金属-有机骨架复合材料(magnetic metal-organic framework, MMOFs) 是磁性纳米粒子与MOFs复 合的一种新型纳米功能材料. 常见的磁性粒子主要有 $\mathrm{Fe}_{3} \mathrm{O}_{4} 、 \gamma-\mathrm{Fe}_{2} \mathrm{O}_{3} 、 \mathrm{Fe} 、 \mathrm{NiO} 、 \mathrm{Co}_{3} \mathrm{O}_{4} 、 \mathrm{MFe}_{2} \mathrm{O}_{4}(\mathrm{M}=\mathrm{Mn}$ 、 $\mathrm{Co} 、 \mathrm{Ni}$ )等, 其中以 $\mathrm{Fe}_{3} \mathrm{O}_{4}$ 作为磁性基体的实例最为常见. 依据磁性粒子与MOFs之间的复合方式, 合成MMOFs 的方法有镶嵌法、叠层法、封装法、混合法 ${ }^{[27]}$. 这种 复合材料集成了磁性材料易分离、易分散、可重复 利用的优点以及MOFs的优异吸附性能, 目前主要应 用于生物医学、环境、催化等领域. MMOFs作为一 种新兴的功能复合材料, 其研究仍处于起步阶段. 目 前, 仅有一篇文章综述了MMOFs的应用 ${ }^{[28]}$, 尚没有针 对环境应用进行专门讨论. 本文分类介绍了 MMOFs 的基本结构, 重点综述了MMOFs在环境领域的应用现 状, 并对MMOFs材料的开发和应用研究作了展望.

\section{MMOFs 结构分类}

根据磁性材料与MOFs的复合方式, 可将报道的 MMOFs 分为以下 4 种: 磁性微球的核壳结构、磁性纳 米颗粒的镶嵌结构、磁性粒子的无规则互混结构、 磁性石墨烯基结构.

\section{1 核壳结构的MMOFs}

核壳结构的纳米复合材料一般由核和包覆在外 边的壳组成, 两者之间通过物理、化学作用相互连接. 由于其独特的物理化学性质, 在许多领域具有潜在的 应用价值; 核壳结构材料一直是纳米复合材料研究的 热点. 近年来, 研究人员致力于开发具有磁性核壳结 构的MOFs材料, 成为一种新的研究趋势. 然而, 目前 有关具有核壳结构的磁性MOFs复合材料的研究尚处 于起步阶段. 典型的MMOFs结构主要包括核@壳和 核@壳@壳等结构.

MMOFs复合材料中核@壳结构是最常见的一类. 其核一般是具有磁性的纳米球, 外壳是多孔的MOFs 层. 这类MMOFs一般是将磁性球表面功能化, 然后分 散于MOFs的前驱体中, 利用层层包裹和自组装的策 略长成核壳结构的MMOFs. 制备过程中, 磁性微球通 过吸附和晶种作用, 调控MOFs 在其表面结晶生长, 最 终形成核壳结构的MMOFs. 磁性微球能调控MOFs 晶 体的生长尺寸, 而外壳的MOFs也能防止磁性微球的 团聚. 相比纯的MOFs, 由于磁性微球的比表面积较低,
复合材料的比表面积会有所下降.

2012年, $\mathrm{Ke}$ 等 ${ }^{[29]}$ 利用层层自组装的方法, 在颈 基乙酸(MAA)处理过的 $\mathrm{Fe}_{3} \mathrm{O}_{4}$ 颗粒表面组装HKUST$1\left[\mathrm{Cu}_{3}(\mathrm{BTC})_{2},(\mathrm{BTC}=\right.$ 均苯三酸)], 成功合成了核壳结构 的 $\mathrm{Fe}_{3} \mathrm{O}_{4} @ \mathrm{HKUST}-1$. 结果表明, 自组装的次数和反应 温度都能影响壳层的厚度; MAA在形成核壳结构的过 程中起到了关键作用. 其形成机理可概括为, $\mathrm{Cu}^{2+}$ 首 先与 $\mathrm{MAA}-\mathrm{Fe}_{3} \mathrm{O}_{4}$ 表面的羧基成键, 然后与配体 $1,3,5$-苯 三甲酸 $\left(\mathrm{H}_{3} \mathrm{BTC}\right)$ 成键. 随后, 许多研究人员利用类似的 方法, 合成了磁性核壳结构的 $\mathrm{Fe}_{3} \mathrm{O}_{4} @ \mathrm{MIL}-100(\mathrm{Fe})^{[30]}$ 、 $\mathrm{Fe}_{3} \mathrm{O}_{4} @ \mathrm{~Tb}-\mathrm{BTC} \mathrm{C}^{[31]}$ 等. 图1是核壳结构MMOFs的合成 示意图及透射电镜图.

2013年, Faustini等 ${ }^{[32]}$ 报道了一种快速合成MOFs 及MOFs复合材料的微流控方法. 他们利用该方法成 功地合成了核壳结构的 $\mathrm{Fe}_{3} \mathrm{O}_{4} @ \mathrm{ZIF}-8$. 具体合成过程 如图2所示, 首先将 $\mathrm{Fe}_{3} \mathrm{O}_{4}$ 的前驱体注入反应器, 在油 相中合成磁性微球; 然后滴入沸石咪唑酯骨架结构 (ZIF-8)的前驱体溶液和表面活性剂聚苯乙烯磺酸钠, 在 $\mathrm{T}$ 管连接处与磁性颗粒分散混合; 最后混合溶液注 入微流反应器中合成外壳ZIF-8. 与传统方法相比, 该 方法通过微流控技术将两步合成反应整合在持续的 流体模式中, 节约了合成时间.

2014年, Zheng课题组 ${ }^{[33]}$ 将合成的 $\mathrm{Fe}_{3} \mathrm{O}_{4}$ 微球加入 到ZIF- 8 的前驱体溶液中, 在 $70^{\circ} \mathrm{C}$ 油浴条件下合成了核 壳结构的 $\mathrm{Fe}_{3} \mathrm{O}_{4} @ \mathrm{ZIF}-8$. 从红外表征推断, 二水合柠檬 酸三钠(合成 $\mathrm{Fe}_{3} \mathrm{O}_{4}$ 的表面活性剂)既可以调控 $\mathrm{Fe}_{3} \mathrm{O}_{4}$ 的 形态, 又可以通过自身羧基改性 $\mathrm{Fe}_{3} \mathrm{O}_{4}$ 的表面. 而表面

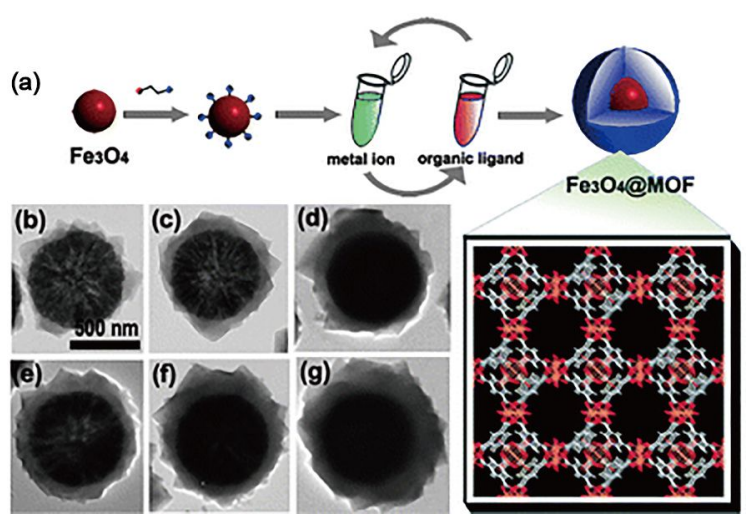

图 $1 \quad \mathrm{Fe}_{3} \mathrm{O}_{4} @\left[\mathrm{Cu}_{3}(\mathrm{BTC})_{2}\right]$ 层层包裹示意图(a)和包裹次数不 同的单个MMOFs的透射电子显微镜图(TEM) $(b \sim g){ }^{[29]}$ (网络 版彩图) 


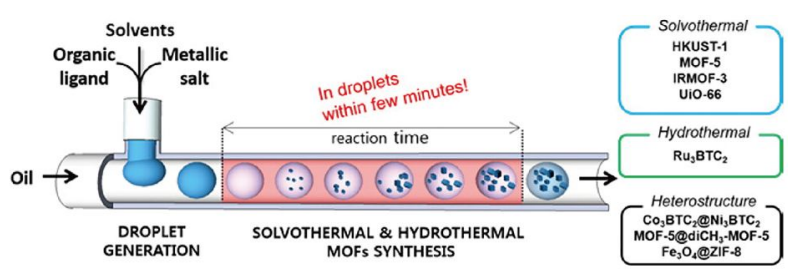

图 2 微流控技术合成 $\mathrm{Fe}_{3} \mathrm{O}_{4} @ \mathrm{ZIF}-8$ 的示意图 ${ }^{[32]}$ (网络版 彩图)

的羧基是ZIF-8生长的关键. 该方法避免了额外表面 活性剂的使用, 并且二水合柠檬酸三钠能有效提高磁 性微球的溶剂分散性, 更有利于MOFs的形成. 表 1 概 括了采用类似方法合成具有核壳结构的MMOFs及其 应用.

除了核@壳结构外, 核@双壳结构的MMOFs也 有报道. 2015年, Huang 等 ${ }^{[39]}$ 在 $\mathrm{Fe}_{3} \mathrm{O}_{4}$ 外边包覆了一层 $\mathrm{SiO}_{2}$, 合成了 $\mathrm{Fe}_{3} \mathrm{O}_{4} @ \mathrm{SiO}_{2}$; 将其加入到HKUST-1 ${ }^{[40]}$ 的前 驱体溶液中, 通过共沉淀方法合成了具有双层壳结构 的 $\mathrm{Fe}_{3} \mathrm{O}_{4} @ \mathrm{SiO}_{2} @ \mathrm{HKUST}-1$. 其合成过程如图3所示.

2015 年, 大连理工大学的 Zhang 课题组 ${ }^{[41]}$ 在 $\mathrm{Fe}_{3} \mathrm{O}_{4} @ \mathrm{SiO}_{2}$ 外表面通过嫁接技术沉积了 Pd纳米颗粒, 再通过层层自组装的策略长成MOFs 外壳. 此类结构 可以有效防止纳米颗粒的团聚和淋出, 确保了贵金属 的稳定存在. 由于高效吸附催化性能和易回收的特 点, 该材料在多相催化和吸附领域具有广阔的应用前 景. 同年, 福州大学 Zhang 等 ${ }^{\left[{ }^{22]}\right]}$ 将 $\mathrm{Fe}_{3} \mathrm{O}_{4} @ \mathrm{SiO}_{2}$ 的壳层 利用3-氨丙基三乙氧基硅烷(APTES)和戊二酸酎羧基 化, 通过液相外延法生成一层UiO-66 (图4) ${ }^{[43]}$. 该材料 可用来富集痕量的软骨藻酸. 相比其他方法, 该方法 具有萃取时间短、检测限低、线性范围宽等优点.

同年, $\mathrm{He}$ 课题 组 ${ }^{[44]}$ 合成了具有双层结构的 $\mathrm{Fe}_{3} \mathrm{O}_{4} @ \mathrm{C} @ \mathrm{ZIF}-8$ (图5). 他们利用水热法合成 $\mathrm{Fe}_{3} \mathrm{O}_{4} @ \mathrm{C}$; 之后, 将其分散于MOFs的前驱体中, 在水浴、搅拌的 条件下合成ZIF-8层. 该合成方法较为简便, 碳层的存 在既可以防止 $\mathrm{Fe}_{3} \mathrm{O}_{4}$ 颗粒的团聚, 又可以充当桥联剂.

2016年, Wehner课题组 ${ }^{[45}$ 将 $\mathrm{Fe}_{3} \mathrm{O}_{4}$ 镶嵌在 $\mathrm{SiO}_{2}$ 基质 中, 利用机械化学改性、溶剂热改性以及原位生长3 种方法, 合成了 $\mathrm{Fe}_{3} \mathrm{O}_{4} / \mathrm{SiO}_{2} @ \mathrm{MOF}$ (图6). 该材料能够 发出冷光, 但与水分子作用后不再具有发光性质. 鉴 于此, 可以作为一种潜在的水分子探测器. 表 2 概括了 采用不同方法合成的具有核双壳结构的MMOFs及其 应用。
表 1 核壳结构的MMOFs及其应用

\begin{tabular}{ccc}
\hline $\mathrm{MMOF}$ & 应用 & Ref. \\
\hline $\mathrm{Fe}_{3} \mathrm{O}_{4} @ \mathrm{HKUST}-1$ & 富集低浓度多肽 & {$[34]$} \\
$\mathrm{Fe}_{3} \mathrm{O}_{4} @ \mathrm{MIL}-100(\mathrm{Fe})$ & 光催化降解染料 & {$[35]$} \\
$\mathrm{Fe}_{3} \mathrm{O}_{4} @ \mathrm{MIL}-100(\mathrm{Fe})$ & 萃取多氯联苯 & {$[36]$} \\
$\mathrm{Fe}_{3} \mathrm{O}_{4} @ \mathrm{~Tb}-\mathrm{BTC}$ & 检测痕量三硝基甲苯(TNT) & {$[31]$} \\
$\mathrm{Fe}_{3} \mathrm{O}_{4} @ \mathrm{ZIF}-8$ & 吸附染料 & {$[33]$} \\
$\mathrm{Fe}_{3} \mathrm{O}_{4} @ \mathrm{MIL}-100(\mathrm{Fe})$ & 选择富集磷酸多肽 & {$[30]$} \\
$\mathrm{Fe}_{3} \mathrm{O}_{4} @ \mathrm{ZIF}-8$ & 富集无机砷 & {$[37]$} \\
$\mathrm{Fe}_{3} \mathrm{O}_{4} @ \mathrm{MIL}-100(\mathrm{Fe})$ & 吸附染料 & {$[38]$} \\
\hline
\end{tabular}

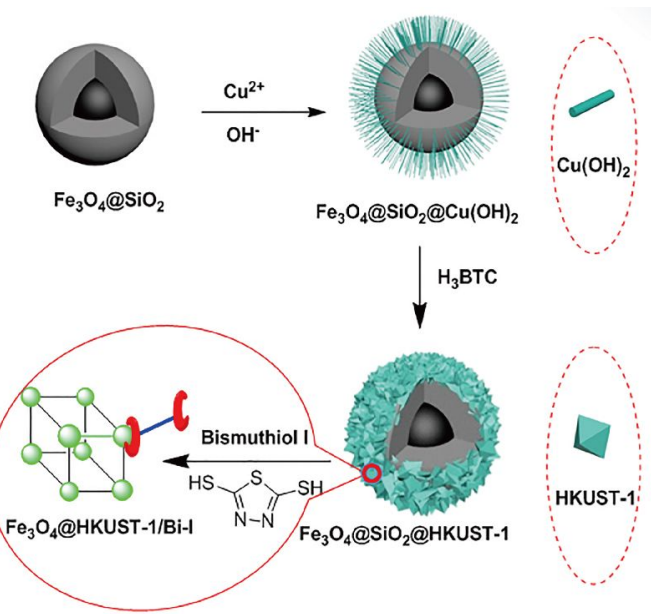

图 $3 \quad \mathrm{Fe}_{3} \mathrm{O}_{4} @ \mathrm{SiO}_{2} @ \mathrm{HKUST}$-1 层层包裹示意图 ${ }^{[39]}$ (网络版 彩图)

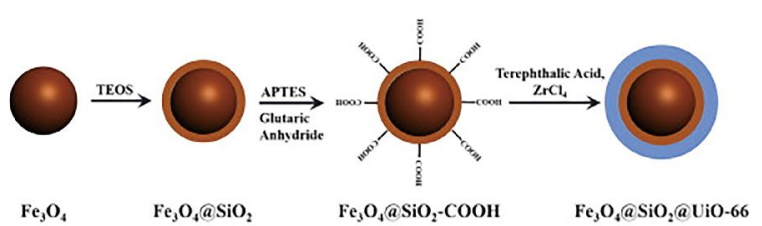

图 $4 \mathrm{Fe}_{3} \mathrm{O}_{4} @ \mathrm{SiO}_{2} @ \mathrm{UiO}-66$ 层层包裹示意图 ${ }^{[42]}$ (网络版彩图)
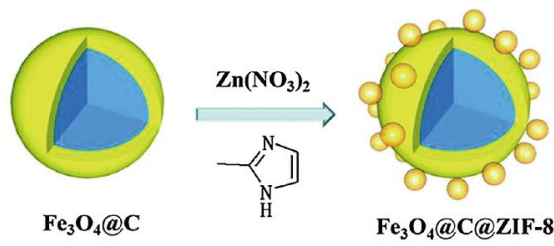

图 $5 \mathrm{Fe}_{3} \mathrm{O}_{4} @ \mathrm{C} @ \mathrm{ZIF}-8$ 结构示意图 ${ }^{[44]}$ (网络版彩图)

\section{2 内镶嵌结构的MMOFs}

核壳型 MMOFs一般是将磁性微球 (约为 400 $500 \mathrm{~nm}$ )经过表面改性后, 在外表面生长MOFs层; 内镶 


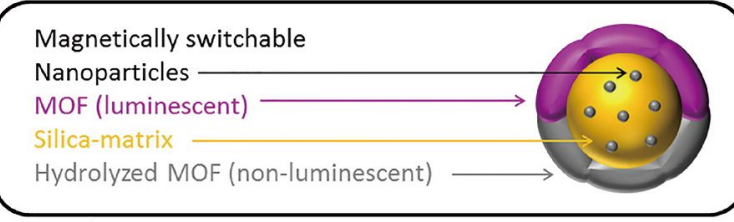

图 $6 \mathrm{Fe}_{3} \mathrm{O}_{4} / \mathrm{SiO}_{2} @ \mathrm{MOF}$ 的结构示意图 ${ }^{[45]}$ (网络版彩图)

表 2 核双壳结构的MMOFs及其应用

\begin{tabular}{ccc}
\hline $\mathrm{MMOF}$ & 应用 & Ref. \\
\hline $\mathrm{Fe}_{3} \mathrm{O}_{4} @ \mathrm{SiO}_{2} @ \mathrm{HKUST}-1$ & 吸附 $\mathrm{Hg}^{2+}$ & {$[39]$} \\
$\mathrm{Fe}_{3} \mathrm{O}_{4} @ \mathrm{SiO}_{2} @ \mathrm{ZIF}-8$ & 催化 & {$[46]$} \\
$\mathrm{Fe}_{3} \mathrm{O}_{4} @ \mathrm{SiO}_{2} @ \mathrm{UiO}-66$ & 萃取软骨藻酸 & {$[42]$} \\
$\mathrm{Fe}_{3} \mathrm{O}_{4} @ \mathrm{C} @ \mathrm{ZIF}-8$ & 药物运输 & {$[44]$} \\
\hline
\end{tabular}

嵌结构的MMOFs 则是在MOFs的晶体表面嵌入磁性 纳米颗粒 (一般粒径几十个纳米). 与 MOFs 晶体尺寸 相比, 磁性纳米颗粒较小, 对MOFs成核过程影响较小, 因此内镶嵌结构的MMOFs最终表现出MOFs的多面体 晶型. 同时, 由于 $\mathrm{Fe}_{3} \mathrm{O}_{4}$ 纳米颗粒的引入, $\mathrm{MOFs}$ 部分孔 径被填充, 最终导致这类复合材料比表面积的下降.

2012年, Lu等 ${ }^{[47}$ 利用聚乙烯吡咯烷酮(PVP)改性 不同形状和尺寸的纳米粒子, 并将其加入到ZIF-8的前 驱体中, 合成了典型的镶嵌纳米粒子的复合结构(图7). 该结构的合成过程, 不是以晶种诱发MOFs的结晶, 而 是依靠MOFs 晶体生长的表面持续不断地吸附纳米颗 粒. 因此, 在MOFs 结晶过程中, 改变磁性纳米颗粒的加 入时间, 可以调控颗粒在MOFs中的空间分布(如图7中 $T_{0}$ 和 $\left.T\right)$.

2014年, Jiang等 ${ }^{[48]}$ 采用相同的策略, 将PVP处理的 $\mathrm{Fe}_{3} \mathrm{O}_{4}$ 纳米颗粒成功镶嵌在ZIF-8中. 在吸附水中羟基 醌的实验中, 表现出超高的吸附性能和良好的磁分离 性质. 2015年, Pang课题组 ${ }^{[49}$ 预先用聚丙烯酸(PPA)处 理 $\mathrm{Fe}_{3} \mathrm{O}_{4}$ 微粒, 使其表面带负电荷, 并将其分散于2-甲 基咪唑的水溶液中, 通过注射原或蠕动洜滴入 $\mathrm{Zn}$ 盐, 室温下在合成ZIF-8 (表面带正电荷)的过程中, 由于静 电作用, $\mathrm{Fe}_{3} \mathrm{O}_{4}$ 微粒嵌入晶体的表面或内部(图8).

\section{3 互混结构的MMOFs (负载、共价键连接、物} 理混合)

与核壳型、镶嵌型结构相比, 混合结构的MMOFs 合成方法比较多. 该类结构的合成方法可以概括为:

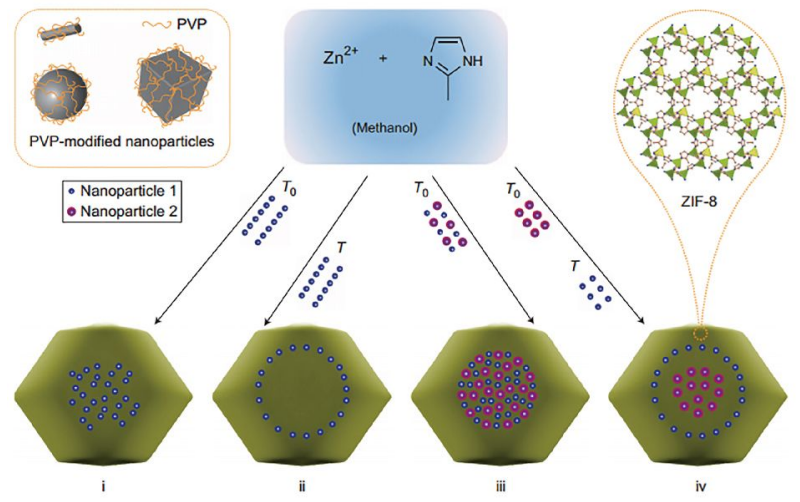

图 7 在ZIF-8 晶体中控制磁性纳米颗粒的嵌入示意图 ${ }^{[47]}$ (网 络版彩图)

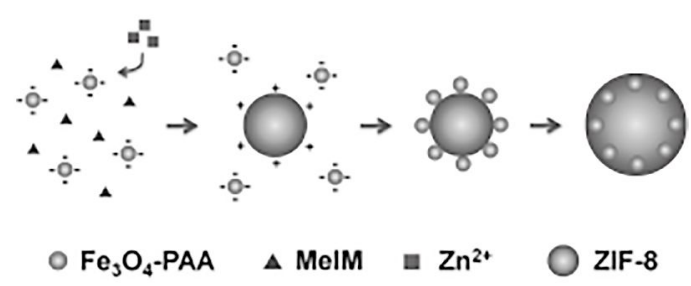

图 8 嵌入型 $\mathrm{Fe}_{3} \mathrm{O}_{4} / \mathrm{ZIF}-8$ 复合材料的合成过程(PAA-聚丙烯 酸 ${ }^{[49]}$

预先合成MOFs, 然后原位合成 $\mathrm{Fe}_{3} \mathrm{O}_{4}$ 磁性颗粒; 或预先 合成 $\mathrm{Fe}_{3} \mathrm{O}_{4}$ 纳米颗粒, 然后化学负载或嫁接到 $\mathrm{MOFs}$; 或 预先分别合成, 再通过物理作用将二者复合. 因此, 互 混结构的MMOFs 都较好地保留了磁性颗粒和MOFs 的形貌. 2014年, Wang 等 ${ }^{[50]}$ 将新制的 $\mathrm{Fe}_{3} \mathrm{O}_{4}$ 与IRMOF-3 的前驱体混合, $90^{\circ} \mathrm{C}$ 回流合成了 $\mathrm{Fe}_{3} \mathrm{O}_{4} / \mathrm{IRMOF}-3$ 复合 材料. 从扫描电子显微镜(SEM)分析可见, IRMOF-3表 面光滑, 而 $\mathrm{Fe}_{3} \mathrm{O}_{4} / \mathrm{IRMOF}-3$ 的表面由于 $\mathrm{Fe}_{3} \mathrm{O}_{4}$ 的负载变 得粗糙. 同时, 复合材料保留了 IRMOF-3 的拓扑形态, 可能由于 $\mathrm{Fe}_{3} \mathrm{O}_{4}$ 磁性颗粒的存在, 晶体明显变小. 随后 许多课题组应用相似的方法, 合成了不同的MMOFs. 主要的研究工作归纳见表3.

2014年, Zhang 等 ${ }^{[56]}$ 研究合成了复合材料 $\mathrm{Fe}_{3} \mathrm{O}_{4} /$ MIL-101(Fe). 他们利用共沉淀方法首先合成 $\mathrm{Fe}_{3} \mathrm{O}_{4}$ 纳 米颗粒, 然后与MIL-101前驱体混合形成同相溶液. 采 用传统水热法合成了具有混合结构的复合材料(图9). 其合成机制为, 共沉淀法合成的 $\mathrm{Fe}_{3} \mathrm{O}_{4}$ 表面含 $-\mathrm{OH}$ 而带 负电荷, 与带正电荷的金属阳离子静电吸引; 然后与 配体 $\mathrm{H}_{2} \mathrm{BDC}(1,4-$ 对苯二甲酸)配位形成MOFs. 该方法 
表 3 混合结构的MMOFs 及其应用

\begin{tabular}{ccc}
\hline MMOFs & 应用 & Ref. \\
\hline $\mathrm{Fe}_{3} \mathrm{O}_{4} / \mathrm{MOF}-5$ & 萃取多氯联苯 & {$[51]$} \\
$\mathrm{Fe}_{3} \mathrm{O}_{4} / \mathrm{HKUST}-1$ & 富集重金属 & {$[52]$} \\
$\mathrm{Fe}_{3} \mathrm{O}_{4} / \mathrm{HKUST}-1$ & 吸附钯 & {$[53]$} \\
$\mathrm{Fe}_{3} \mathrm{O}_{4} / \mathrm{HKUST}-1$ & 富集重金属 & {$[54]$} \\
$\mathrm{Fe}_{3} \mathrm{O}_{4} / \mathrm{MIL}-101$ & 萃取重金属 & {$[55]$} \\
\hline
\end{tabular}

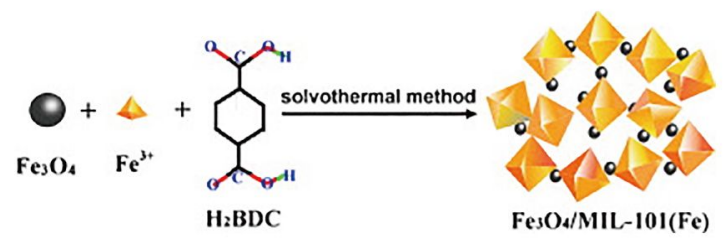

图 $9 \mathrm{Fe}_{3} \mathrm{O}_{4} / \mathrm{MIL}-101(\mathrm{Fe})$ 的合成示意图 ${ }^{[56]}$ (网络版彩图)

无需对 $\mathrm{Fe}_{3} \mathrm{O}_{4}$ 颗粒进行化学改性, 直接混合, 合成简单, 适合较大规模合成.

2016年, Jiang课题组 ${ }^{[57]}$ 将预先合成的MIL-101(Cr) 加入到 $\mathrm{Fe}_{3} \mathrm{O}_{4}$ 的前驱体中, 然后利用多相共沉淀法原 位合成 $\mathrm{Fe}_{3} \mathrm{O}_{4}$. 如图10所示, 从 SEM和TEM图可以看出, $\mathrm{Fe}_{3} \mathrm{O}_{4}$ 纳米微粒成功地负载于MIL-101 $(\mathrm{Cr})$ 的外表面, 实 现了磁功能化.

除了通过化学方法合成MMOFs 外, Pino 课题 组 ${ }^{[58]}$ 利用简单的物理混合法实现了 $\mathrm{Fe}_{3} \mathrm{O}_{4}$ 与HKUST-1 的复合。该方法十分简单, 首先分别合成 $\mathrm{Fe}_{3} \mathrm{O}_{4}$ 和 HKUST-1, 然后将二者混合进行涡流处理, 确保形成 $\mathrm{Fe}_{3} \mathrm{O}_{4} / \mathrm{HKUST}-1$ 复合材料. 最后将其用于磁辅助-固相 微萃取水中的多环芳烃, 结果表明, 该材料不仅富集 效果好, 而且能够较好地实现磁性分离.

\section{4 磁性石墨烯基结构MOFs}

近年来, 二维材料凭借其独特的性质受到了广泛 的关注 ${ }^{[59,60]}$. 以石墨烯为代表的二维材料与MOFs的复 合也有报道. 由于石墨烯的表面具有许多含氧官能团, 因此可以通过多种方法进行改性, 同时也利于MOFs 的生长. 在磁性石墨烯基结构MOFs复合材料中, 石墨 烯充当了磁性粒子与MOFs的结构导向剂, 也起到防 止磁性粒子团聚的作用. 同时, 由于 MOFs的引入, 复 合材料保留了较大的比表面积. 这种多功能的复合材 料在吸附、分离等领域显示了优越的性能.

2014年, Huang 等 ${ }^{[61]}$ 通过一种简易的方法合成了
一系列三元复合材料, 他们在 $\mathrm{MoS}_{2}$ 纳米片、氧化石墨 烯、还原石墨烯的表面嵌入了 $\mathrm{Pt}$ 金属纳米颗粒, 并在 二维材料的边缘生长了一层ZIF-8. 2015年, Wang 等 ${ }^{[2]}$ 利用类似的方法合成了磁性石墨烯(maG)@PDA@ZrMOFs (图11), 并利用该材料有效萃取水中的双酚A. 2016年, Lin课题组 ${ }^{[63]}$ 先合成磁性石墨烯, 并将其加 入到ZIF-67的前驱体中, 制备出二维磁性石墨烯负载 ZIF-67的复合材料(图12).

\section{MMOFs 在环境中的应用}

磁性MOFs集成了MOFs和磁性材料的众多优点. 这种复合材料可以通过外部磁场的作用实现分离, 大 大简化了繁琐的分离过程, 并且具有易回收利用的特 性, 是一种具有潜在价值的绿色固体吸附材料. 在环 境分析和环境修复领域中, 具有广阔的应用前景.
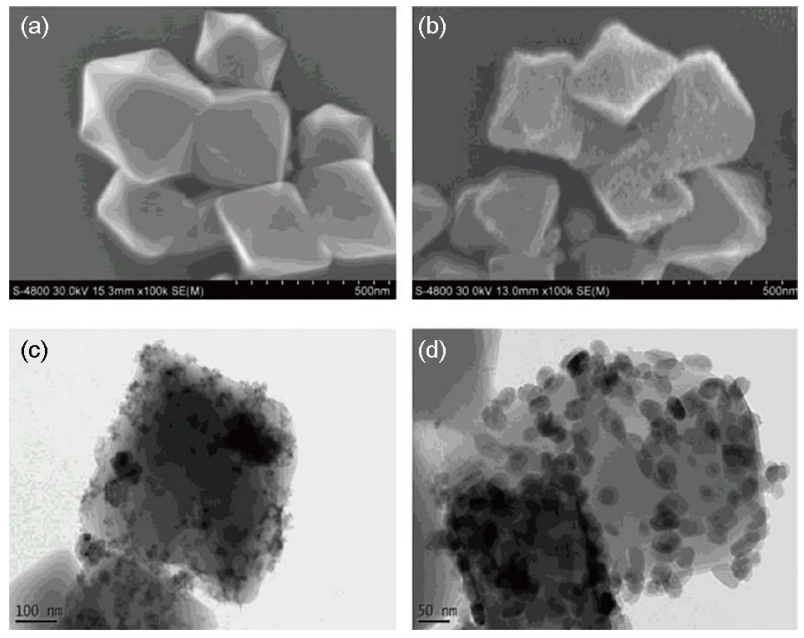

图 10 MIL-101 (a)和复合材料 $\mathrm{Fe}_{3} \mathrm{O}_{4} / \mathrm{HKUST}-1$ 的SEM/TEM $(b \sim d)^{[57]}$

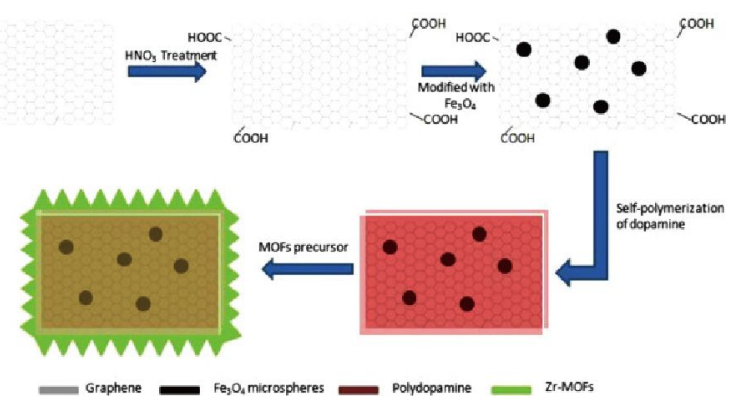

图 11 maG@PDA@Zr-MOFs的合成过程 ${ }^{[62]}$ (网络版彩图) 


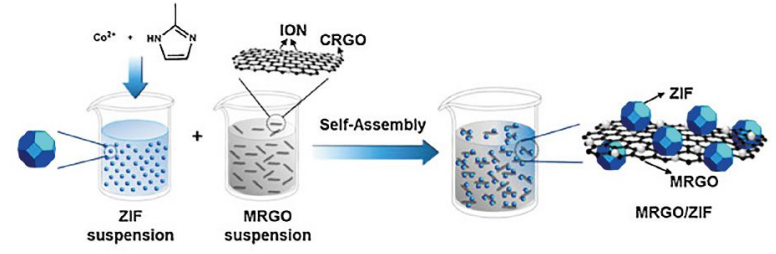

图 12 ZIF-67/MRGO的自组装示意图 ${ }^{[63]}$ (网络版彩图)

\section{1 环境分析}

\subsection{1 重金属的富集和检测}

重金属离子是水中常见的有毒污染物,多数金属 离子对人类健康有重大威胁. 然而, 对水中痕量离子的 富集和检测是目前环境分析的一大难点和热点. 将磁 性MOFs应用于固相萃取中, 取得了引人瞩目的效果.

Bagheri课题组 ${ }^{[53]}$ 利用吡啶衍生物通过回流方法 对 $\mathrm{Fe}_{3} \mathrm{O}_{4}$ 进行改性, 在外表面生长HKUST-1, 合成了核 壳结构的磁性 $\mathrm{Fe}_{3} \mathrm{O}_{4}-\mathrm{Py} @$ @)HKUST-1. 从实际的鱼类、 沉淀物、水、土壤等样品中萃取痕量金属钯离子, 结合火焰原子吸收光谱测定. 结果显示, 该方法具 有较好的选择性和灵敏度, 建立了从不同的实际基 质中富集-分析痕量钯离子的新方法. 相对 $\mathrm{Fe}_{3} \mathrm{O}_{4}-\mathrm{Py}$, $\mathrm{Fe}_{3} \mathrm{O}_{4}-\mathrm{Py} @ H K U S T-1$ 具有较高的吸附容量; 原因可能 与MOFs比表面积大以及合适的孔径有关, $\mathrm{Fe}_{3} \mathrm{O}_{4}$-Py在 复合材料中不仅提供了磁分离的性能, 同时吡啶功能
团的改性也提高了对钯的选择. Sohrabi课题组 ${ }^{[64]}$ 通过 类似的方法合成了同种核壳结构的MMOFs, 并用于萃 取镉离子和铅离子. 对于两种离子的吸附量约为 190 $\mathrm{mg} / \mathrm{g}$. 该材料成功应用于实际水样、鱼类等样品中富 集两种离子.

Wang 课题 组 ${ }^{[52]}$ 将合成的 $\mathrm{Fe}_{3} \mathrm{O}_{4}$ 颗粒, 加入到 $\mathrm{Cu}_{3}(\mathrm{BTC})_{2}$ 的前驱体中, 合成 $\mathrm{Fe}_{3} \mathrm{O}_{4} / \mathrm{Cu}_{3}(\mathrm{BTC})_{2}$ 复合材 料, 利用双硫腙对合成的复合材料进行后期改性. 该 材料被用于富集水中痕量的铅离子. 研究表明, 双硫 腙改性的MMOFs 对 $\mathrm{Pb}^{2+}$ 具有较高的吸附能力, 研究者 认为, 铅离子与双硫腙具有较强的鳌合作用. 该方法 具有较低的检测限, 吸附量达 $1.67 \mathrm{mg} / \mathrm{g}$. 之后, 该课题 组又对 $\mathrm{Fe}_{3} \mathrm{O}_{4} / \mathrm{Cu}_{3}(\mathrm{BTC})_{2}$ 复合材料进行颈基改性, 用于 萃取不同食物样品中的铅离子; 他们对萃取铅离子的 方法进行了归纳分析, 从数据可以看出, 该方法具有 最低的检测限和最大的吸附容量. 这归因于MOFs 较 大的比表面积和表面的自由-SH基团. 以上两个实例 中, 功能基团都是嫁接在MOFs的开放金属位点上,而 这些功能基团是复合材料对 $\mathrm{Pb}^{2+}$ 选择性和亲和性提高 的直接原因, 因此 $\mathrm{Fe}_{3} \mathrm{O}_{4}$ 在复合材料中只起到了磁分离 的作用. Taghizadeh课题组 ${ }^{[65]}$ 做了类似的研究工作. 他 们将合成的复合材料用于富集水中的 4 种金属离子, 同时也通过实验设计优化了萃取分析条件. 表4 (重金 属)、表5 (有机物)归纳了MMOFs主要的应用。

表 4 MMOFs在分析中的应用(富集重金属离子)

\begin{tabular}{|c|c|c|c|c|c|c|}
\hline MMOFs & 分析物 & 洗脱液 & 线性范围(ng/mL) & 检测 限 (ng/mL) & $\mathrm{RSD} \%$ & Ref. \\
\hline $\mathrm{Fe}_{3} \mathrm{O}_{4} @ \mathrm{ZIF}-8$ & As & $\mathrm{HCl}$ & - & 0.006 & 1.9 & [37] \\
\hline $\mathrm{Fe}_{3} \mathrm{O}_{4} / \mathrm{IRMOF}-3$ & $\mathrm{Cu}^{2+}$ & $\mathrm{NaCl}$ & $0.1 \sim 10$ & 0.073 & 0.4 & {$[50]$} \\
\hline $\mathrm{Fe}_{3} \mathrm{O}_{4} / \mathrm{Cu}_{3}(\mathrm{BTC})_{2}-\mathrm{H}_{2} \mathrm{Dz}$ & $\mathrm{Pb}^{2+}$ & $\mathrm{HNO}_{3}$ & $0.01 \sim 5$ & 0.0046 & 3.2 & {$[52]$} \\
\hline $\mathrm{Fe}_{3} \mathrm{O}_{4} @ \mathrm{ED} @ \mathrm{MIL}-101(\mathrm{Fe})$ & $\begin{array}{l}\mathrm{Cd}(\mathrm{II}), \\
\mathrm{Pb}(\mathrm{II}), \\
\mathrm{Zn}(\mathrm{II}) \\
\mathrm{Cr}(\mathrm{III})\end{array}$ & $\mathrm{EDTA}+\mathrm{HNO}_{3}$ & $\begin{array}{l}0.5 \sim 100 \\
2.5 \sim 250 \\
0.6 \sim 120 \\
1.5 \sim 150\end{array}$ & $\begin{array}{c}0.15 \\
0.8 \\
0.2 \\
0.5\end{array}$ & $\begin{array}{c}7 \\
4.9 \\
6.8 \\
5.5\end{array}$ & {$[55]$} \\
\hline$\left(\mathrm{Fe}_{3} \mathrm{O}_{4}\right.$-Pyridine $) / \mathrm{Cu}_{3}(\mathrm{BTC})_{2}$ & $\operatorname{Pd}(\mathrm{II})$ & $\mathrm{K}_{2} \mathrm{SO}_{4}+\mathrm{NaOH}$ & $10^{3} \sim 10^{5}$ & 0.37 & 2.1 & [53] \\
\hline $\mathrm{SH}-\mathrm{Fe}_{3} \mathrm{O}_{4} / \mathrm{Cu}_{3}(\mathrm{BTC})_{2}$ & $\mathrm{~Pb}(\mathrm{II})$ & $\mathrm{HNO}_{3}$ & $1 \sim 20$ & 0.29 & 2.4 & {$[54]$} \\
\hline $\mathrm{Fe}_{3} \mathrm{O}_{4}$-pyridine- $\mathrm{Cu}_{3}(\mathrm{BTC})_{2}$ & $\begin{array}{l}\mathrm{Cd}(\mathrm{II}) \\
\mathrm{Pb}(\mathrm{II})\end{array}$ & $\mathrm{EDTA}+\mathrm{NaOH}$ & $\begin{array}{c}1 \sim 80 \\
2.5 \sim 125\end{array}$ & $\begin{array}{c}0.21 \\
1.1\end{array}$ & $\begin{array}{l}3.6 \\
4.3\end{array}$ & [64] \\
\hline $\mathrm{Fe}_{3} \mathrm{O}_{4}$-Dithizone- $\mathrm{Cu}_{3}(\mathrm{BTC})_{2}$ & $\begin{array}{l}\mathrm{Cd}(\mathrm{II}) \\
\mathrm{Zn}(\mathrm{II}) \\
\mathrm{Ni}(\mathrm{II}) \\
\mathrm{Pb}(\mathrm{II})\end{array}$ & Thiourea $+\mathrm{NaOH}$ & $\begin{array}{l}1 \sim 110 \\
1 \sim 120 \\
2 \sim 135 \\
4 \sim 140\end{array}$ & $\begin{array}{c}0.12 \\
0.39 \\
0.98 \\
1.2\end{array}$ & $\begin{array}{l}3.6 \\
4.3 \\
4.7 \\
3.9\end{array}$ & {$[65]$} \\
\hline $\begin{array}{l}\mathrm{Fe}_{3} \mathrm{O}_{4} @ 2,5 \text {-dimercapto-1,3,4- } \\
\text { thiadiazole } \mathrm{Cu}_{3}(\mathrm{BTC})_{2}\end{array}$ & $\begin{array}{l}\mathrm{Cd}(\mathrm{II}) \\
\mathrm{Zn}(\mathrm{II}) \\
\mathrm{Pb}(\mathrm{II}) \\
\end{array}$ & EDTA & $\begin{array}{c}0.4 \sim 75 \\
0.5 \sim 100 \\
2.5 \sim 250\end{array}$ & $\begin{array}{l}0.10 \\
0.15 \\
0.75 \\
\end{array}$ & $\begin{array}{l}6.8 \\
8.3 \\
5.4 \\
\end{array}$ & {$[66]$} \\
\hline
\end{tabular}


表 5 MMOFs在分析中的应用(富集有机物)

\begin{tabular}{|c|c|c|c|c|c|c|}
\hline MMOFs & 分析物 & 洗脱液 & 线性范围 $(\mathrm{ng} / \mathrm{mL})$ & 检测限 $(\mathrm{ng} / \mathrm{L})$ & $\mathrm{RSD} \%$ & Ref. \\
\hline $\mathrm{Fe}_{3} \mathrm{O}_{4} @$ HKUST-1 & 多环芳烃(PAHs) & 乙腈 & $\begin{array}{l}0.01 \sim 0.75 \\
0.01 \sim 0.75 \\
0.01 \sim 0.75 \\
0.01 \sim 0.75 \\
0.01 \sim 0.75 \\
0.01 \sim 0.75 \\
0.01 \sim 0.75 \\
0.05 \sim 0.75\end{array}$ & $\begin{array}{l}1.8 \\
0.8 \\
1.8 \\
1.3 \\
4.6 \\
1.3 \\
1.2 \\
1.2\end{array}$ & $\begin{array}{c}8.7 \\
7.6 \\
4.6 \\
6.5 \\
2.6 \\
10 \\
11 \\
9.0\end{array}$ & {$[58]$} \\
\hline $\mathrm{Fe}_{3} \mathrm{O}_{4} @ \mathrm{MIL}-100(\mathrm{Fe})$ & 多氯联苯(PCBs) & 超纯水/正己烷 & $0.05 \sim 4$ & $1.07 \sim 1.57$ & $2.05 \sim 11.92$ & {$[36]$} \\
\hline $\mathrm{Fe}_{3} \mathrm{O}_{4} @ \mathrm{SiO}_{2} @ \mathrm{UiO}-66$ & 软骨藻酸 & 乙酸/乙腈 & $0.002 \sim 1$ & 1.45 & $\leqslant 5.0$ & {$[42]$} \\
\hline Magnetic MOF-5 & $\begin{array}{l}\text { PAHs } \\
\text { 赤霉酸 }\end{array}$ & 乙腈/甲酸 & $\begin{array}{c}0.005 \sim 0.5 \\
0.03 \sim 100 \\
0.50 \sim 100 \\
0.02 \sim 60 \\
0.12 \sim 140\end{array}$ & $\begin{array}{c}0.91 \sim 1.96 \\
6 \sim 80\end{array}$ & $1.7 \sim 9.7$ & {$[67]$} \\
\hline $\mathrm{Fe}_{3} \mathrm{O}_{4} @ \mathrm{SiO}_{2}-\mathrm{MIL}-101$ & 6种PAHs & 乙腈 & $\begin{array}{c}0.01 \sim 250 \\
0.1 \sim 250 \\
0.01 \sim 250 \\
0.01 \sim 250 \\
0.1 \sim 250 \\
0.05 \sim 250\end{array}$ & $\begin{array}{c}2.8 \\
15.0 \\
5.5 \\
3.9 \\
27.2 \\
9.8\end{array}$ & $\begin{array}{l}4.3 \\
3.1 \\
6.4 \\
4.8 \\
8.7 \\
7.1\end{array}$ & {$[68]$} \\
\hline magG@PDA@Zr-MOFs & $\begin{array}{c}5 \text { 种双酚 } \\
(\mathrm{BPs})\end{array}$ & 乙腈 & $\begin{array}{c}50 \sim 20000 \\
50 \sim 20000 \\
50 \sim 20000 \\
200 \sim 500000 \\
500 \sim 50000\end{array}$ & $\begin{array}{c}100 \\
1000 \\
1000 \\
1000 \\
1000\end{array}$ & $\begin{array}{l}0.62 \\
2.62 \\
2.86 \\
4.89 \\
4.47\end{array}$ & {$[62]$} \\
\hline $\mathrm{Fe}_{3} \mathrm{O}_{4} @ \mathrm{MIL}-100(\mathrm{Cr})$ & 孔雀绿(MG) & 甲醇 & $40 \sim 2000$ & 12 & 3.5 & [69] \\
\hline $\mathrm{Fe}_{3} \mathrm{O}_{4}-\mathrm{MOF}-5(\mathrm{Fe})$ & PCBs & 正已烷 & $0.01 \sim 500$ & $0.061 \sim 1$ & - & {$[51]$} \\
\hline
\end{tabular}

最近, Zou课题组 ${ }^{[37]}$ 应用简单的层层自组装策略, 合成了具有核壳结构的 $\mathrm{Fe}_{3} \mathrm{O}_{4} @ \mathrm{ZIF}-8$. 该新型材料被 用来富集水中的无机砷离子, 并通过原子苂光光谱 (AFS) 测定. 他们得出的结论是, $\mathrm{Fe}_{3} \mathrm{O}_{4} @ Z$ ZIF- 8 与ZIF-8 具有相近的吸附效果, 而远高于 $\mathrm{Fe}_{3} \mathrm{O}_{4}$ 纳米颗粒, $\mathrm{Fe}_{3} \mathrm{O}_{4}$ 纳米颗粒只是为核壳材料提供了磁性, 并没有对无机 砷的吸附产生显著影响.

\subsection{2 有机物的富集和检测}

除重金属离子外, 水中的有机污染物不仅种类较 多, 而且毒性较大. 按照常规的分类方法, 有机污染物 可以分为持久性污染物 $(\mathrm{POPs})$ 、环境激素类 $(\mathrm{EDCs})$ 、 药品和个人护理品 (PPCPs)、有机大分子染料等. 因 此富集、分离、检测水中的有机污染物具有重要意 义. 目前, 许多课题组利用MMOFs作为富集材料, 开 展了重要的实验研究.

2012 年, Huo 课题 ${ }^{{ }^{[}{ }^{[68]}}$ 报道了磁性复合材料 $\left(\mathrm{Fe}_{3} \mathrm{O}_{4} @ \mathrm{SiO}_{2}-\mathrm{MIL}-101\right)$ 用于固相萃取水中的多环芳
烃. 他们首先合成了 $\mathrm{Fe}_{3} \mathrm{O}_{4} @ \mathrm{SiO}_{2}$ 和 MIL-101, 在超 声辅助下, 磁性颗粒在MOFs 外表面附着, 最后合成 了 $\mathrm{Fe}_{3} \mathrm{O}_{4} @ \mathrm{SiO}_{2}-\mathrm{MIL}-101$. 研究表明, $\mathrm{Fe}_{3} \mathrm{O}_{4} @ \mathrm{SiO}_{2}$ 与 MIL-101之间存在的静电作用力是形成复合材料的 关键因素. 利用 $\mathrm{Fe}_{3} \mathrm{O}_{4} @ \mathrm{SiO}_{2}-\mathrm{MIL}-101$ 富集, 结合高效 液相色谱对实际水体中的多环芳烃进行了分析. 结 果表明, 该方法具有吸附剂用量少、进样量少和检 测限低的优点.

2013年, $\mathrm{Hu}$ 课题组 ${ }^{[67]}$ 将氨基功能化的 $\mathrm{Fe}_{3} \mathrm{O}_{4}$, 通 过共价键的作用力连接在MOF-5 的外表面, 合成了 $\mathrm{MOF}-5 / \mathrm{NH}_{2}-\mathrm{Fe}_{3} \mathrm{O}_{4}$ 磁性复合材料. 该磁性复合材料用 于富集不同极性的多环芳烃. 结果表明, 该材料对极 性芳烃类物质具有较强的吸附效果, 而 $\pi-\pi$ 键的相互 作用和氢键的形成是高亲和力的重要因素. 他们利 用MOF-5/ $\mathrm{NH}_{2}-\mathrm{Fe}_{3} \mathrm{O}_{4}$ 富集不同样品中痕量的多环芳烃, 通过气相色谱/质谱联用测定含量. 同时, 也可以利用 该材料富集赤霉酸并结合 LC/MS/MS进行分析.

由以上两个实例分析可得: MMOFs对PAHs有较 
好的富集效果, 主要是由于MOFs与 $\mathrm{PAHs}$ 间存在 $\pi-\pi$ 相 互作用及氢键作用, 而复合材料中的 $\mathrm{Fe}_{3} \mathrm{O}_{4}$ 颗粒主要发 挥了磁性分离的作用.

\section{2 环境修复}

水体中的污染物主要有重金属离子、无机阴离 子和有机物等. 水体环境修复是指通过利用化学、物 理、生物等技术去除这些污染物, 从而达到净化水质 的目的. 传统的修复技术包括混凝、沉淀、过滤、吸 附、降解等. 凭借快速分离的优势, 并结合高比表面 积、均一孔径、易修饰等特点, MMOFs作为一种高 效吸附剂和催化剂, 对于水环境的原位修复具有重要 作用.

\subsection{1 吸附去除污染物}

吸附技术是基于吸附质和吸附剂之间亲和力, 去 除污染物的一项技术. 该技术具有应用范围广、低能 耗、易操作、低污染、可再生等优点, 是实现环境修 复的重要手段之一. MMOFs具备了MOFs疏松多孔、 比表面积大的优点, 同时具备磁性性能, 因此普遍应 用于吸附领域. 在去除水中染料、重金属离子、其他 新型有机污染物方面发挥着重要作用.

有机染料是水中重要的污染物, 因此去除染料,
对实现环境修复具有重要意义. MMOFs用于去除 染料的报道相对较多. 2014年, Li课题组 ${ }^{[70]}$ 合成了 复合材料 $\mathrm{Fe}_{3} \mathrm{O}_{4} / \mathrm{HKUST}-1-\mathrm{GO}$, 并将其用于吸附水中 的甲基蓝(MB). 实验结果表明, 氧化石墨烯(GO)对 $\mathrm{Fe}_{3} \mathrm{O}_{4} / \mathrm{HKUST}-1$ 的结构有重大影响. GO的加入使比表 面积和孔径大大减小, 但对 $\mathrm{MB}$ 的吸附容量增大了. 同 时, 经过 5 次循环, 复合材料对 $\mathrm{MB}$ 的去除率仍保持在 $90 \%$ 以上. 由此可见, 复合材料 $\mathrm{Fe}_{3} \mathrm{O}_{4} / \mathrm{HKUST}-1-\mathrm{GO}$ 是 一种良好的去除MB的吸附剂. 同年, Dadfarnia课题 组 ${ }^{[71]}$ 合成了核壳型的 $\mathrm{Fe}_{3} \mathrm{O}_{4} / \mathrm{MIL}-100(\mathrm{Fe})$ 复合材料. 相 比各种活性炭、改性沸石等材料, 该复合材料对于甲 基红的吸附饱和量提高了 3 倍以上. 2015年, Xiong课 题组 ${ }^{[72]}$ 成功合成了 $\gamma-\mathrm{Fe}_{2} \mathrm{O}_{3} / \mathrm{C} @ H K U S T-1$ 复合材料. 对 于MB的最大吸附量达 $101.4 \mathrm{mg} / \mathrm{g}$. 表6归纳了MMOFs 用于去除重金属和染料的主要工作.

重金属离子是水中另一类重要的污染物. 目前, MMOFs应用于去除重金属离子的报道相对较少. 2015 年, Ricco课题组 ${ }^{[26]}$ 合成的铁氧化物/MIL-53(A1)复合材 料用于吸附水中的 $\mathrm{Pb}^{2+}$. 研究表明, 氨基化的有机配体 提高了复合材料对 $\mathrm{Pb}^{2+}$ 吸附能力. 由于路易斯碱 $\left(\mathrm{NH}_{2}-\right)$ 能够与路易斯酸 $\left(\mathrm{Pb}^{2+}\right)$ 发生强烈的配位作用, 使得复合 材料对 $\mathrm{Pb}^{2+}$ 的饱和吸附量高达 $492.4 \mathrm{mg} / \mathrm{g}$. 图13为不同 种类MOFs 对 $\mathrm{Pb}^{2+}$ 的吸附效果柱状图.

表 6 MMOFs去除重金属和染料

\begin{tabular}{|c|c|c|c|c|c|c|}
\hline MMOFs & 污染物 & $\operatorname{BET}\left(\mathrm{m}^{2} / \mathrm{g}\right)$ & $Q_{\max }(\mathrm{mg} / \mathrm{g})$ & 动力学模型 & 等温线模型 & Ref. \\
\hline $\mathrm{Fe}_{3} \mathrm{O}_{4} @ \mathrm{SiO}_{2} @ \mathrm{HKUST-1/Bi-I}$ & $\mathrm{Hg}^{2+}$ & 67.43 & 264 & 假二级模型 & Langmuir & [39] \\
\hline$\gamma-\mathrm{Fe}_{2} \mathrm{O}_{3} / \mathrm{C} @$ HKUST-1 & $\mathrm{Cr}_{2} \mathrm{O}_{7}^{2-}$ & 993.4 & 101.4 & 假二级模型 & Langmuir & [72] \\
\hline Magnetic MIL-53(Al)-NH ${ }_{2}$ & $\mathrm{~Pb}^{2+}$ & 120 & 492.4 & - & Freundlich & [26] \\
\hline $\mathrm{Fe}_{3} \mathrm{O}_{4} / \mathrm{ZIF}-8$ & 羟基醌 & 1394 & 2527 & 假二级模型 & Langmuir & [48] \\
\hline 磁性石墨烯/ZIF-67 & 苯并三唑 & - & 300 & 假二级模型 & Freundlich & [73] \\
\hline $\mathrm{Fe}_{3} \mathrm{O}_{4} @ \mathrm{ZIF}-8$ & 亚甲基蓝 & 1068 & 20.2 & - & - & [33] \\
\hline $\mathrm{Fe}_{3} \mathrm{O}_{4} / \mathrm{HKUST}-1-\mathrm{GO}$ & 亚甲基蓝 & 533 & - & 假一级模型 & Langmuir & [70] \\
\hline $\mathrm{Fe}_{3} \mathrm{O}_{4} / \mathrm{HKUST}-1$ & 亚甲基蓝 & 79.52 & 245 & 假二级模型 & Freundlich & [74] \\
\hline $\mathrm{Fe}_{3} \mathrm{O}_{4} @ \mathrm{MIL}-100(\mathrm{Fe})$ & 罗丹明B & 206.14 & 28.90 & 假二级模型 & Freundlich & [75] \\
\hline $\mathrm{Fe}_{3} \mathrm{O}_{4} @ \mathrm{MIL}-100(\mathrm{Fe})$ & 亚甲基蓝 & 366.14 & 73.8 & 假二级模型 & Langmuir & [38] \\
\hline 磁性石墨烯/ZIF-67 & 孔雀绿 & - & 2682 & 假二级模型 & $\begin{array}{l}\text { Langmuir- } \\
\text { Freundlich }\end{array}$ & [63] \\
\hline $\mathrm{Fe}_{3} \mathrm{O}_{4} @ \mathrm{MIL}-100(\mathrm{Cr})$ & $\begin{array}{c}\text { 甲基橙 } \\
\text { 甲酚橙 } \\
\text { 苂光素钠 }\end{array}$ & 3300 & - & 假二级模型 & Langmuir & {$[57]$} \\
\hline $\mathrm{Fe}_{3} \mathrm{O}_{4} @ \mathrm{MIL}-100(\mathrm{Cr})$ & $\begin{array}{l}\text { 酸红 } \\
\text { 橙黄 } \mathrm{G}\end{array}$ & 1790 & $\begin{array}{l}142.9 \\
200.0\end{array}$ & 假二级模型 & Langmuir & [76] \\
\hline$\gamma-\mathrm{Fe}_{2} \mathrm{O}_{3} / \mathrm{C} @$ HKUST-1 & 甲基蓝 & 993.4 & 370.2 & 假二级模型 & Langmuir & [72] \\
\hline
\end{tabular}




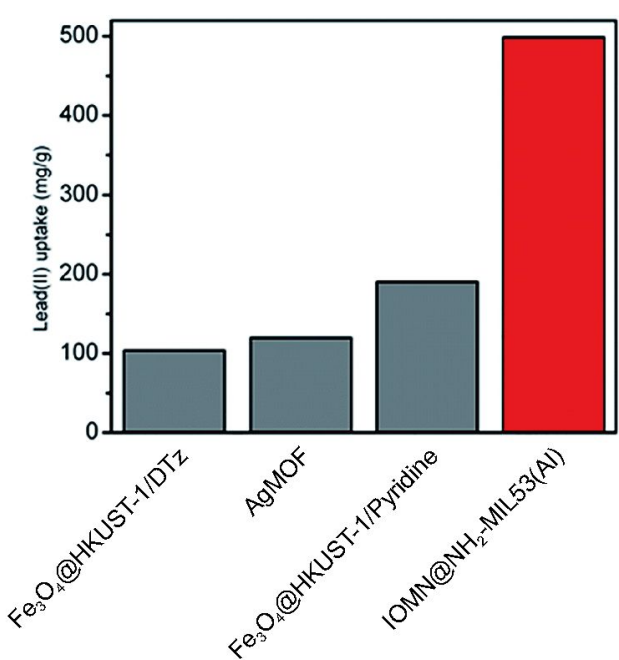

图 13 不同 MOFs 对 $\mathrm{Pb}^{2+}$ 的吸附效果 ${ }^{[20]}$ (网络版彩图)

2015 年, Huang 课题组 ${ }^{[39]}$ 报道了 $\mathrm{Fe}_{3} \mathrm{O}_{4} @ \mathrm{SiO}_{2} @$ HKUST-1复合材料. 他们利用后期改性策略, 将铋试剂 处理过的复合材料用于去除水中的 $\mathrm{Hg}^{2+}$. 结果表明, 铋 试剂功能化的复合材料对 $\mathrm{Hg}^{2+}$ 的去除率高达 $99 \%$. 数 据显示, 该复合材料具有较宽的 $\mathrm{pH}$ 应用范围 $(\mathrm{pH}=2 \sim 9$, 图14(a)), 而且去除率接近 $100 \%$. 然而 $\mathrm{Fe}_{3} \mathrm{O}_{4} @ \mathrm{SiO}_{2}$ 与 $\mathrm{Fe}_{3} \mathrm{O}_{4} @ \mathrm{SiO}_{2} @ \mathrm{HKUST}-1$ 均对 $\mathrm{Hg}^{2+}$ 几乎没有吸附效果. 从图14(b)可以看出, 铋试剂功能化的复合材料对 $\mathrm{Hg}^{2+}$ 的吸附具有较高的选择性. 除 $\mathrm{Pb} 、 \mathrm{Cr}$ 有较小干扰外, $\mathrm{Cd} 、 \mathrm{Co} 、 \mathrm{Mn} 、 \mathrm{Ni} 、 \mathrm{Zn}$ 离子无明显竞争作用. 这是因 为铋试剂中的颈基基团与 $\mathrm{Hg}^{2+}$ 具有较强的亲和力.

除染料和重金属离子外, 新型的有机污染物由于 其高毒性、持久性、难降解等特性, 近年来, 引起了 研究者的关注. 目前, MMOFs 用于去除水中新型污 染物的研究也见诸报道. 2015年, Jiang课题组 ${ }^{[48]}$ 利用 $\mathrm{Fe}_{3} \mathrm{O}_{4} / \mathrm{ZIF}-8$ 复合材料, 去除水中的羟基醌. 吸附动力 学研究表明, 在100 200 min 内可快速达到平衡, 并且 吸附容量达 $2527 \mathrm{mg} / \mathrm{g}$. 同时, 研究人员也分析了吸附 羟基醌的可能机制. 在实验条件下, 吸附剂和吸附质 不带有高的电荷, 羟基醌是以分子而非酚盐的形式被 吸附. 因此, 静电作用不是主要的吸附作用力, 而咪唑 环和芳香环之间的 $\pi-\pi$ 键和氢键的作用是吸附过程的 主要机制, 如图15所示.

2016年, Lin 课题组 ${ }^{[73]}$ 研究了 ZIF-MG (magnetic graphene) 吸附水中的苯并吡唑(BTA), 研究表明, ZIF-MG对BTA的最大吸附量为 $183 \mathrm{mg} / \mathrm{g}$; 而ZIF-67、
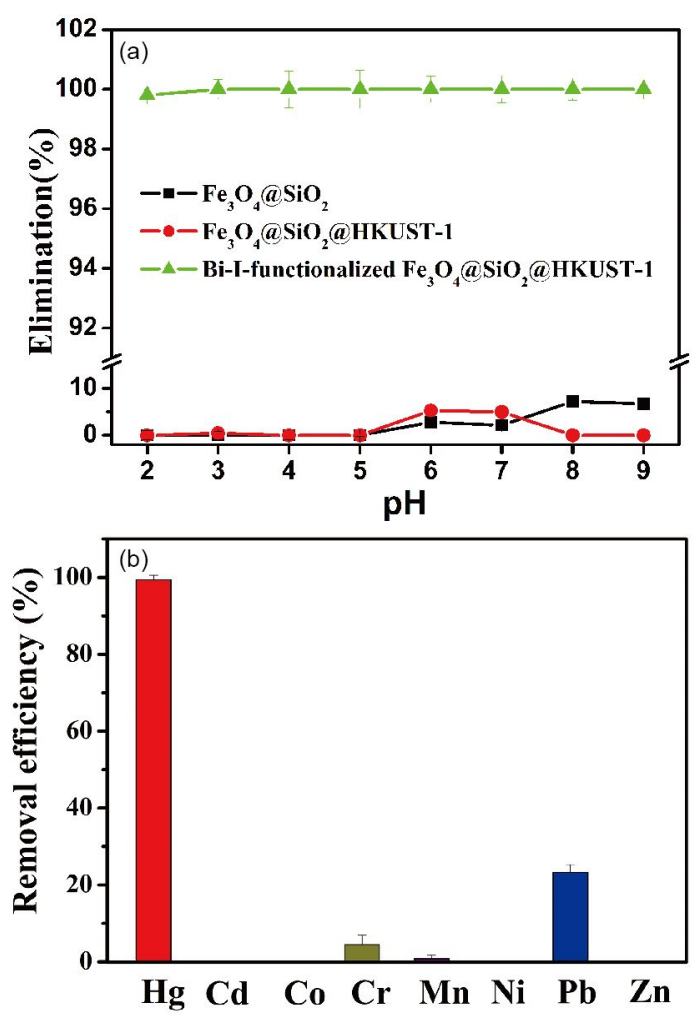

图 $14 \mathrm{Fe}_{3} \mathrm{O}_{4} @ \mathrm{SiO}_{2} @$ HKUST-1复合材料对 $\mathrm{Hg}^{2+}$ 的吸附 ${ }^{[39]}$. (a) 不同 $\mathrm{pH}$ 的吸附效果; (b) 吸附去除 $\mathrm{Hg}^{2+}$ 的选择实验(网络 版彩图)

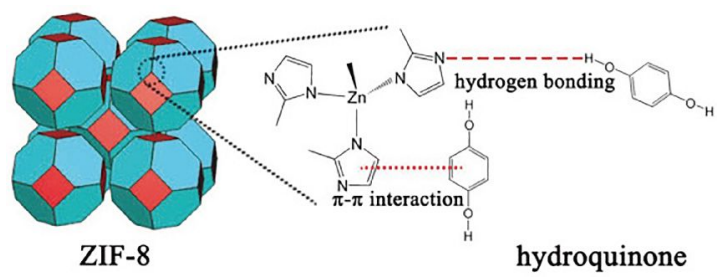

图 $15 \mathrm{Fe}_{3} \mathrm{O}_{4}-\mathrm{ZIF}-8$ 复合材料对羟基醌的吸附机制 ${ }^{[48]}$ (网络 版彩图)

MG、物理混合物(1:1)的吸附量分别为23、163、122 $\mathrm{mg} / \mathrm{g}$ (图16(a)). 他们认为, ZIF-MG复合材料吸附量提 高的原因是自组装过程使得ZIF-67的分散性更好, 从 而产生了协同作用. 如图16(b)所示, 复合材料在较宽 的 $\mathrm{pH}$ 范围内具有较高的吸附量; 当 $\mathrm{pH}<3$ 或 $\mathrm{pH}>9$ 时, 由 于复合材料与BTA带有同种电荷, 静电斥力导致吸附 量有所下降.

\subsection{2 催化降解污染物}

催化降解是一种有效的环境修复技术. 随着新的 

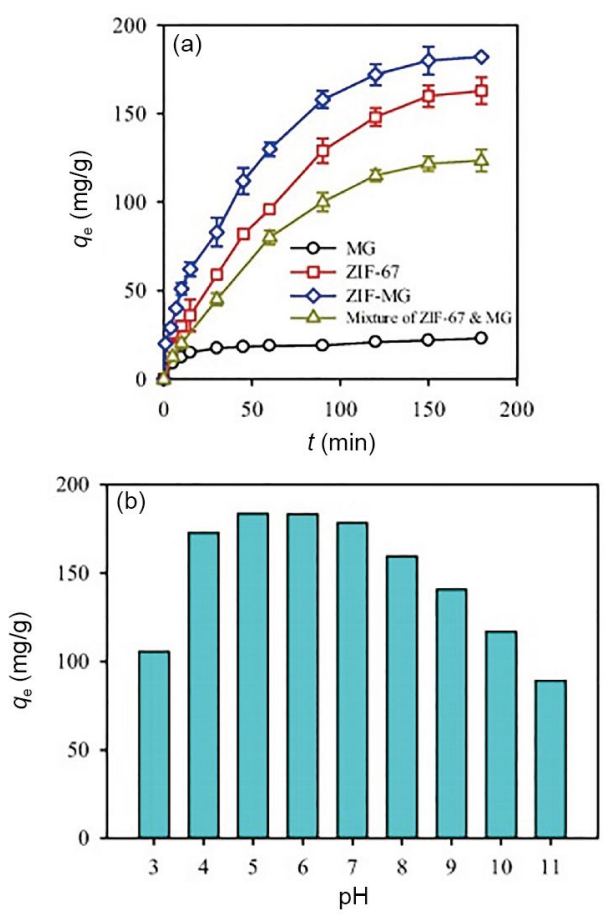

图 16 ZIF-MG复合材料对BTA的吸附. (a) 吸附动力学; (b) $\mathrm{pH}$ 的影响 ${ }^{[73]}$ (网络版彩图)

难降解污染物的不断出现, 开发具有更强分解能力、 更快降解速率的催化剂成为当前的研究热点之一. MMOFs 具有较大的比表面积和均一的孔径, 同时可 以简易地实现磁性分离. 在降解的过程中, 既可以提 高较多的活性位点, 又可以解决回收利用的问题. 因 此在降解污染物, 实现原位水修复领域具有广阔的前 景. 目前, 有关MMOFs催化降解水中污染物的报道仅

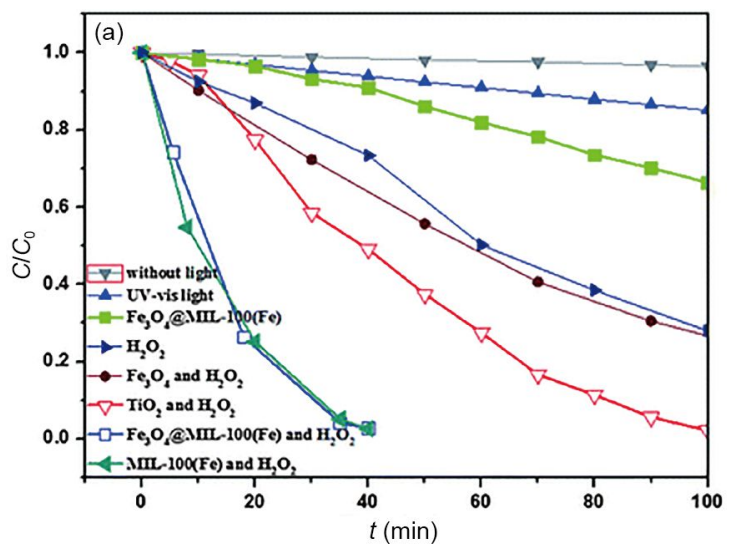

有几篇.

2013年, Zhang课题组 ${ }^{[35]}$ 利用 $\mathrm{Fe}_{3} \mathrm{O}_{4} / \mathrm{MIL}-100(\mathrm{Fe})$ 光 催化降解甲基蓝, 并与商业 $\mathrm{TiO}_{2}$ 和 $\mathrm{C}_{3} \mathrm{~N}_{4}$ 做了比较研 究. 结果表明, 相同实验条件下, $\mathrm{Fe}_{3} \mathrm{O}_{4} / \mathrm{MIL}-100(\mathrm{Fe})$ 在紫外可见光的照射下, $40 \mathrm{~min}$ 内降解率达 $99 \%$, 而 商业 $\mathrm{TiO}_{2}$ 只有 $52 \%$; 其降解常数是 $\mathrm{TiO}_{2}$ 的 3 倍. 在可见 光的照射下, $200 \mathrm{~min}$ 内 $\mathrm{Fe}_{3} \mathrm{O}_{4} / \mathrm{MIL}-100(\mathrm{Fe})$ 降解率高达 $99.77 \%$, 而 $\mathrm{C}_{3} \mathrm{~N}_{4}$ 仅有 $10 \%$; 降解常数是 $\mathrm{C}_{3} \mathrm{~N}_{4}$ 的 33 倍. 此 外, $\mathrm{Fe}_{3} \mathrm{O}_{4} / \mathrm{MIL}-100(\mathrm{Fe})$ 与 MIL-100( $\left.\mathrm{Fe}\right)$ 的催化性能也做 了比较, 数据显示(图17(a)), 二者具有相近的催化效率, 但前者可以磁性分离, 重复利用. 重复5次, 降解效果 没有明显改变, 如图 17(b)所示. 该实例中, $\mathrm{Fe}_{3} \mathrm{O}_{4}$ 仅赋 予复合材料磁分离的性质, 并没有提高材料的催化效 果, 而MIL-100 $(\mathrm{Fe})$ 才是复合材料中主要的活性组分.

2015年, Ke课题组 ${ }^{[77]}$ 合成了核壳结构的复合材料 $\mathrm{Au}-\mathrm{Fe}_{3} \mathrm{O}_{4} @ \mathrm{MIL}-100(\mathrm{Fe})$, 用于催化还原4-硝基苯酚的 反应. 这种复合结构使贵金属 $(\mathrm{Au})$ 纳米颗粒分散程 度更高, 稳定性更好, 提高了催化剂的活性和稳定性. 研究显示, MIL-100(Fe)的催化效率较低, 而复合材料 $\mathrm{Au}-\mathrm{Fe}_{3} \mathrm{O}_{4} @ \mathrm{MIL}-100(\mathrm{Fe})$ 的催化性能明显增加, 但随着 自组装包裹次数 (5、10、20、30次)的增多, 催化性能 呈下降趋势. 从图18(a)中可以看出自组装5次的催化 效果最佳. 原因可能是当复合材料的壳层太厚时, 反应 前驱体的扩散阻力也随之增大, 从而限制了催化效果. 循环性能是催化剂的一个重要指标. 如图18(b)所示, 虽然复合材料的循环性能动力学参数明显降低, 但 5 次 循环的催化效果只降低了 $20 \%$. 相比裸露的 $\mathrm{Au}-\mathrm{Fe}_{3} \mathrm{O}_{4}$, 由于MIL-100(Fe)壳层的保护机制, 使得复合材料具有

图 17 不同条件下的降解效果(a)和循环效果 $(b)^{[35]}$ (网络版彩图)

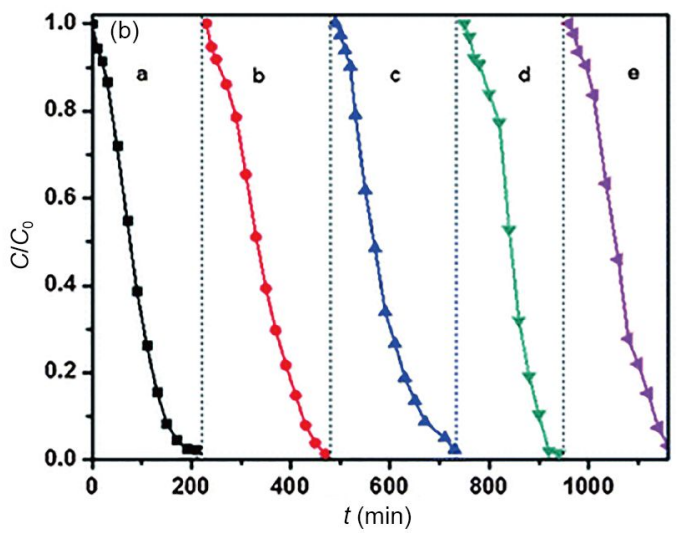



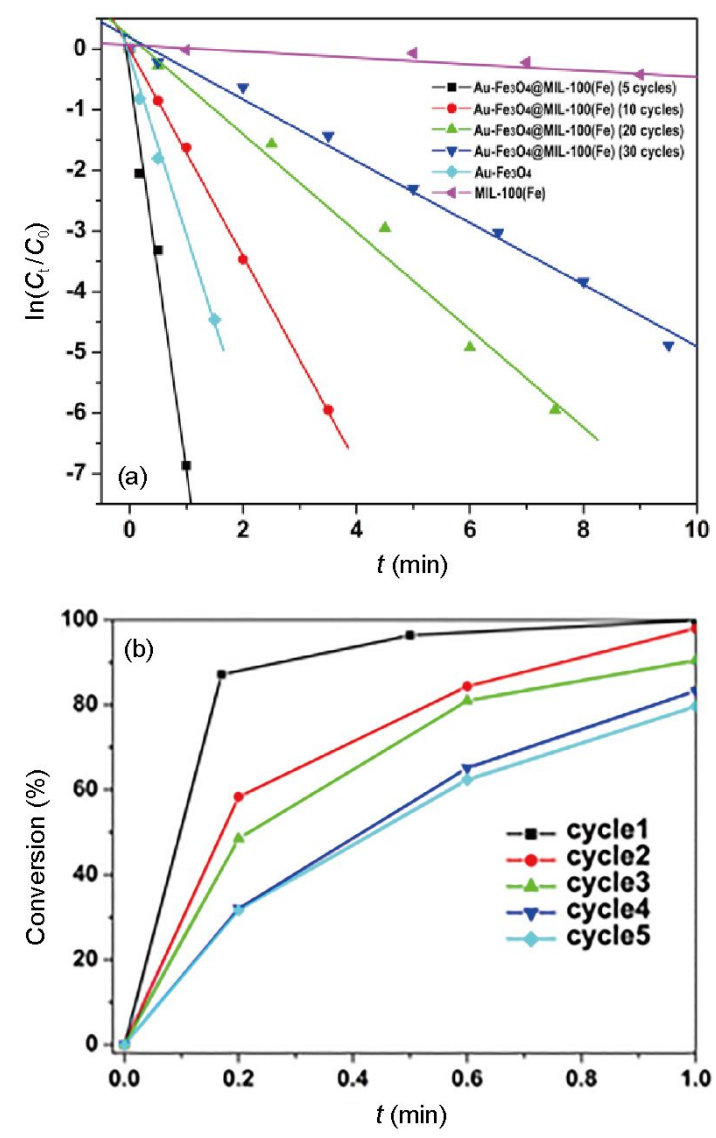

图 $18 \mathrm{Au}-\mathrm{Fe}_{3} \mathrm{O}_{4} @ \mathrm{MIL}-100(\mathrm{Fe})$ 的降解效果 (a)和循环效果 (b) ${ }^{[77]}$ (网络版彩图)

了良好的循环催化性能.

2015 年，Zeng 等 ${ }^{[78]}$ 合成了核壳结构的 $\mathrm{Co}_{3} \mathrm{O}_{4} @ \mathrm{MOFs}$, 利用过硫酸盐高级氧化法降解4-氯酚. 该方法在 $60 \mathrm{~min}$ 内使4-氯酚的降解率高达 $100 \%$. 复 合材料的降解机制如图19所示: 多孔MOFs的强吸附

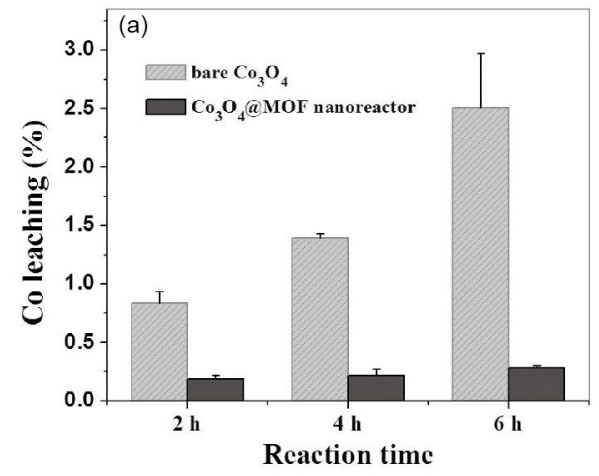

图 $20 \mathrm{Co}_{3} \mathrm{O}_{4} @ \mathrm{MOF}$ s 的稳定性和循环性能
作用, 能够将溶液主体中的4-氯酚和过硫酸盐富集到 $\mathrm{Co}_{3} \mathrm{O}_{4}$ 的表面; 狭小的MOFs 孔洞使得反应物的浓度 瞬间增大, 同时在 $\mathrm{Co}_{3} \mathrm{O}_{4}$ 的活化下, 又有大量自由基 产生, 从而有效地促进了污染物的降解. 复合材料的 催化性能来自于 $\mathrm{Co}_{3} \mathrm{O}_{4}$. 同时, 由于壳层 $\mathrm{MOFs}$ 的存 在, 降低了Co离子的溶出, 避免了二次污染(图20(a)). 图20(b)表明, 该催化剂循环4次, 4-氯酚的降解率仍能 保持 $100 \%$. 复合材料中, $\mathrm{Co}_{3} \mathrm{O}_{4}$ 不仅可以高效活化过 硫酸盐, 同时又赋予复合材料磁分离的性能. 因此, 该 复合材料是一种良好的降解污染物的催化剂.

\section{4 结论与展望}

磁性金属有机骨架材料是纳米复合材料的前沿方 向之一, 在实际应用方面显示了显著的优势. 按磁性 材料与MOFs材料的复合方式, 可将磁性MOFs 材料分 为4类: 典型的MOFs包裹磁性微球的核壳结构、磁性 纳米颗粒嵌入MOFs 结构、MOFs 与磁性粒子的互混 结构、磁性石墨烯基MOFs. 该类复合材料既集成了 MOFs的高吸附性能和易改性的特点, 又具备了磁性

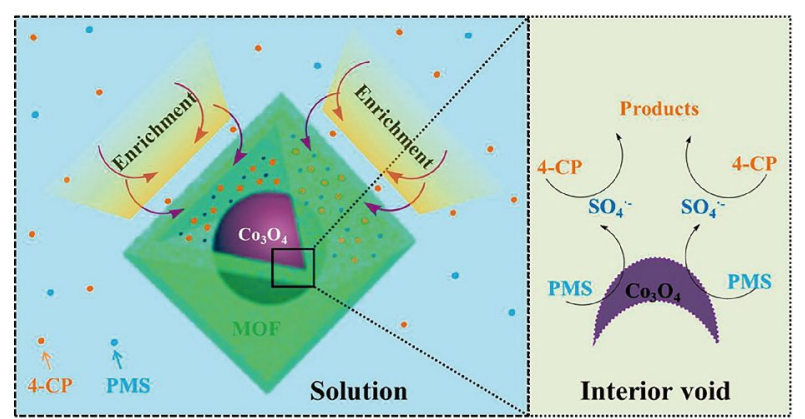

图 194 -氯酚的降解机制(网络版彩图)

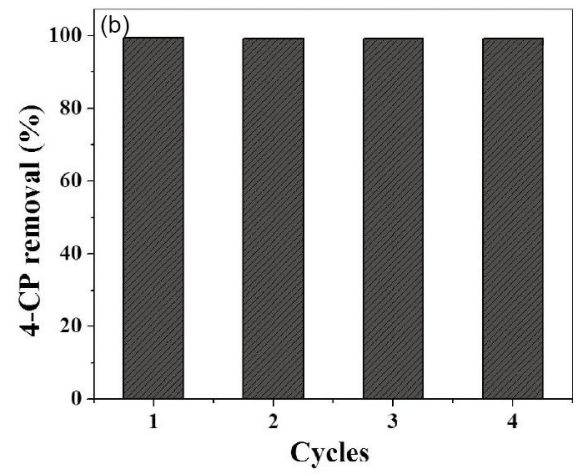


材料易分离的优点. MMOFs 在环境领域的应用已成 为新的研究热点. 目前, 在水环境分析和环境修复领 域的应用相对较少, 深入的研究有待进一步开发.

磁性金属有机骨架复合材料的基础研究尚处于 起步阶段, 研究人员在材料的构建和应用方面取得了 一定的进展. 但合成材料的过程也面临 4 个问题. (1) 核壳型MMOFs: 由于磁性微球与MOF的结合力较弱, 需要层层自组装; 整个合成过程不仅耗时、繁琐, 并 且浪费大量原材料. (2) 在合成MMOFs的过程中, 需要 用到表面活性剂, 而大多数表面活性剂属于有机物质,
易造成二次污染. (3) 文献中只有少数种类的 MOFs用 于制备MMOFs, 如ZIF-8、ZIF-67、MIL-101 $(\mathrm{Cr} / \mathrm{Fe})$ 、 HKUST-1、UiO-66等. (4) 当前MMOFs的基础研究数 据贵乏, 无法推广至工程应用.

综上所述, 现阶段应以MOFs的制备为基础, 进一 步开发更多种类的MMOFs, 同时调控复合材料的综 合性能以提高环境修复和环境分析的效能. 研究开发 经济、高效、简易的, 不使用有机表面活性剂的绿色 合成途径, 对于拓展MMOFs的应用范围具有重要的 意义.

\section{参考文献}

1 Farrusseng D, Aguado S, Pinel C. Angew Chem Int Ed, 2009, 48: 7502-7513

2 Colombo V, Galli S, Choi HJ, Han GD, Maspero A, Palmisano G, Masciocchi N, Long JR. Chem Sci, 2011, 2: 1311-1319

3 Férey G, Mellot-Draznieks C, Serre C, Millange F, Dutour J, Surblé S, Margiolaki I. Science, 2005, 309: 2040-2042

4 Ramsahye NA, Trung TK, Bourrelly S, Yang Q, Devic T, Maurin G, Horcajada P, Llewellyn PL, Yot P, Serre C, Filinchuk Y, Fajula F, Férey G, Trens P. J Phys Chem C, 2011, 115: 18683-18695

5 Murray LJ, Dincă M, Long JR. Chem Soc Rev, 2009, 38: 1294-1314

6 Kreno LE, Leong K, Farha OK, Allendorf M, Van Duyne RP, Hupp JT. Chem Rev, 2012, 112: 1105-1125

7 Horcajada P, Gref R, Baati T, Allan PK, Maurin G, Couvreur P, Férey G, Morris RE, Serre C. Chem Rev, 2012, 112: 1232-1268

8 Lykourinou V, Chen Y, Wang XS, Meng L, Hoang T, Ming LJ, Musselman RL, Ma S. J Am Chem Soc, 2011, 133: 10382-10385

9 Liu Y, Gao P, Huang C, Li Y. Sci China Chem, 2015, 58: 1553-1560

0 Ge D, Lee HK. J Chromatogr A, 2011, 1218: 8490-8495

1 Wang C, Liu X, Keser Demir N, Chen JP, Li K. Chem Soc Rev, 2016, 45: 5107-5134

Cohen SM. Chem Sci, 2010, 1: 32-36

Wang S, Morris W, Liu Y, McGuirk CM, Zhou Y, Hupp JT, Farha OK, Mirkin CA. Angew Chem Int Ed, 2015, 54: 14738-14742

Gui B, Zhang YF, Meng XS, Wang C. Sci Sin Chim, 2016, 46: 472-477

Zhu QL, Xu Q. Chem Soc Rev, 2014, 43: 5468-5512

Hong DY, Hwang YK, Serre C, Férey G, Chang JS. Adv Funct Mater, 2009, 19: 1537-1552

Zhang Q, Yu J, Cai J, Song R, Cui Y, Yang Y, Chen B, Qian G. Chem Commun, 2014, 50: 14455-14458

Zhang JY, Zhang N, Zhang L, Fang Y, Deng W, Yu M, Wang Z, Li L, Liu X, Li J. Sci Rep, 2015, 5: 13514

Yee KK, Reimer N, Liu J, Cheng SY, Yiu SM, Weber J, Stock N, Xu Z. J Am Chem Soc, 2013, 135: 7795-7798

Wang Y, Ye G, Chen H, Hu X, Niu Z, Ma S. J Mater Chem A, 2015, 3: 15292-15298

Liu Y, Tang Z. Adv Mater, 2013, 25: 5819-5825

Falcaro P, Ricco R, Yazdi A, Imaz I, Furukawa S, Maspoch D, Ameloot R, Evans JD, Doonan CJ. Coordin Chem Rev, 2016, 307: 237-254

Jahan M, Bao Q, Loh KP. J Am Chem Soc, 2012, 134: 6707-6713

Yang SJ, Choi JY, Chae HK, Cho JH, Nahm KS, Park CR. Chem Mater, 2009, 21: 1893-1897

Xiang Z, Hu Z, Cao D, Yang W, Lu J, Han B, Wang W. Angew Chem Int Ed, 2011, 50: 491-494

Ricco R, Konstas K, Styles MJ, Richardson JJ, Babarao R, Suzuki K, Scopece P, Falcaro P. J Mater Chem A, 2015, 3: 19822-19831

王瑞莹, 张超艳, 王淑萍, 周友亚. 化学进展, 2015, 27: 945-952

Ricco R, Malfatti L, Takahashi M, Hill AJ, Falcaro P. J Mater Chem A, 2013, 1: 13033-13045

Ke F, Qiu LG, Yuan YP, Jiang X, Zhu JF. J Mater Chem, 2012, 22: 9497-9500

Chen Y, Xiong Z, Peng L, Gan Y, Zhao Y, Shen J, Qian J, Zhang L, Zhang W. ACS Appl Mater Interf, 2015, 7: 16338-16347

Qian JJ, Qiu LG, Wang YM, Yuan YP, Xie AJ, Shen YH. Dalton Trans, 2014, 43: 3978-3983

Faustini M, Kim J, Jeong GY, Kim JY, Moon HR, Ahn WS, Kim DP. J Am Chem Soc, 2013, 135: 14619-14626 
Zheng J, Cheng C, Fang WJ, Chen C, Yan RW, Huai HX, Wang CC. CrystEngComm, 2014, 16: 3960-3964

Zhao M, Deng C, Zhang X, Yang P. Proteomics, 2013, 13: 3387-3392

Zhang CF, Qiu LG, Ke F, Zhu YJ, Yuan YP, Xu GS, Jiang X. J Mater Chem A, 2013, 1: 14329-14334

Chen X, Ding N, Zang H, Yeung H, Zhao RS, Cheng C, Liu J, Chan TWD. J Chromatogr A, 2013, 1304: 241-245

Zou Z, Wang S, Jia J, Xu F, Long Z, Hou X. Microchem J, 2016, 124: 578-583

Shao Y, Zhou L, Bao C, Ma J, Liu M, Wang F. Chem Eng J, 2016, 283: 1127-1136

Huang L, He M, Chen B, Hu B. J Mater Chem A, 2015, 3: 11587-11595

Nie M, Lu S, Li Q, Liu XW, Du SJ. Sci Sin Chim, 2016, 46: 357-364

Zhang T, Lin L, Zhang X, Liu H, Yan X, Qiu J, Yeung KL. Mater Lett, 2015, 148: 17-21

Zhang W, Yan Z, Gao J, Tong P, Liu W, Zhang L. J Chromatogr A, 2015, 1400: 10-18

Bai Y, Dou Y, Xie LH, Rutledge W, Li JR, Zhou HC. Chem Soc Rev, 2016, 45: 2327-2367

He M, Zhou J, Chen J, Zheng F, Wang D, Shi R, Guo Z, Wang H, Chen Q. J Mater Chem B, 2015, 3: 9033-9042

Wehner T, Mandel K, Schneider M, Sextl G, Müller-Buschbaum K. ACS Appl Mater Interf, 2016, 8: 5445-5452

Li Q, Jiang S, Ji S, Ammar M, Zhang Q, Yan J. J Solid State Chem, 2015, 223: 65-72

Lu G, Li S, Guo Z, Farha OK, Hauser BG, Qi X, Wang Y, Wang X, Han S, Liu X, DuChene JS, Zhang H, Zhang Q, Chen X, Ma J, Loo SCJ, Wei WD, Yang Y, Hupp JT, Huo F. Nat Chem, 2012, 4: 310-316

Jiang X, Chen HY, Liu LL, Qiu LG, Jiang X. J Alloys Compd, 2015, 646: 1075-1082

Pang F, He M, Ge J. Chem Eur J, 2015, 21: 6879-6887

Wang Y, Xie J, Wu Y, Hu X. Microchim Acta, 2014, 181: 949-956

Lin S, Gan N, Qiao L, Zhang J, Cao Y, Chen Y. Talanta, 2015, 144: 1139-1145

Wang Y, Xie J, Wu Y, Ge H, Hu X. J Mater Chem A, 2013, 1: 8782-8789

Bagheri A, Taghizadeh M, Behbahani M, Akbar Asgharinezhad A, Salarian M, Dehghani A, Ebrahimzadeh H, Amini MM. Talanta, 2012, 99: 132-139

Wang Y, Chen H, Tang J, Ye G, Ge H, Hu X. Food Chem, 2015, 181: 191-197

Babazadeh M, Hosseinzadeh-Khanmiri R, Abolhasani J, Ghorbani-Kalhor E, Hassanpour A. RSC Adv, 2015, 5: 19884-19892

Zhang S, Jiao Z, Yao W. J Chromatogr A, 2014, 1371: 74-81

Jiang Z, Li Y. J Taiwan Inst Chem E, 2016, 59: 373-379

Rocío-Bautista P, Pino V, Ayala JH, Pasán J, Ruiz-Pérez C, Afonso AM. J Chromatogr A, 2016, 1436: 42-50

Li S, Yang K, Tan C, Huang X, Huang W, Zhang H. Chem Commun, 2016, 52: 1555-1562

Sun B, Mo Y, Wang L, Yue J, Wang D. Sci Sin Chim, 2016, 46: 994-1006

Huang X, Zheng B, Liu Z, Tan C, Liu J, Chen B, Li H, Chen J, Zhang X, Fan Z, Zhang W, Guo Z, Huo F, Yang Y, Xie LH, Huang W, Zhang H. ACS Nano, 2014, 8: 8695-8701

Wang X, Deng C. Talanta, 2015, 144: 1329-1335

Lin KYA, Lee WD. Appl Surf Sci, 2016, 361: 114-121

Sohrabi MR, Matbouie Z, Asgharinezhad AA, Dehghani A. Microchim Acta, 2013, 180: 589-597

Taghizadeh M, Asgharinezhad AA, Pooladi M, Barzin M, Abbaszadeh A, Tadjarodi A. Microchim Acta, 2013, 180: 1073-1084

Ghorbani-Kalhor E, Hosseinzadeh-Khanmiri R, Babazadeh M, Abolhasani J, Hassanpour A. Can J Chem, 2015, 93: 518-525

Hu Y, Huang Z, Liao J, Li G. Anal Chem, 2013, 85: 6885-6893

Huo SH, Yan XP. Analyst, 2012, 137: 3445-3451

Maya F, Palomino Cabello C, Estela JM, Cerdà V, Turnes Palomino G. Anal Chem, 2015, 87: 7545-7549

Li L, Liu XL, Gao M, Hong W, Liu GZ, Fan L, Hu B, Xia QH, Liu L, Song GW, Xu ZS. J Mater Chem A, 2014, 2: 1795-1801

Dadfarnia S, Haji Shabani AM, Moradi SE, Emami S. Appl Surf Sci, 2015, 330: 85-93

Xiong Y, Ye F, Zhang C, Shen S, Su L, Zhao S. RSC Adv, 2015, 5: 5164-5172

Lin KYA, Lee WD. Chem Eng J, 2016, 284: 1017-1027

Zhao X, Liu S, Tang Z, Niu H, Cai Y, Meng W, Wu F, Giesy JP. Sci Rep, 2015, 5: 11849

Liu H, Ren X, Chen L. J Indust Eng Chem, 2016, 34: 278-285

Wang T, Zhao P, Lu N, Chen H, Zhang C, Hou X. Chem Eng J, 2016, 295: 403-413

Ke F, Wang L, Zhu J. Nanoscale, 2015, 7: 1201-1208

Zeng T, Zhang X, Wang S, Niu H, Cai Y. Environ Sci Technol, 2015, 49: 2350-2357 


\title{
The fabrication of magnetic metal-organic frameworks composites and their application in environment
}

\author{
Jiangbo Huo ${ }^{1,2}$, Haojie Cui ${ }^{1}$, Minglai $\mathrm{Fu}^{1 *}$, Baoling Yuan ${ }^{3 *}$ \\ ${ }^{1}$ Key Laboratory of Urban Pollutant Conversion, Institute of Urban Environment, Chinese Academy of Sciences, Xiamen 361021, China \\ ${ }^{2}$ University of Chinese Academy of Sciences, Beijing 100049, China \\ ${ }^{3}$ College of Civil Engineering, Huaqiao University, Xiamen 361020, China \\ *Corresponding authors (email: mlfu@iue.ac.cn; blyuan@hqu.edu.cn)
}

\begin{abstract}
The magnetic metal-organic frameworks (MMOFs), as one of novel functional materials, were constructed by combining the magnetism of magnetic particles with the uniform pore sizes, large surface areas, excellent chemical and thermostability of meal-organic frameworks to enhance their performance as potential catalysts and adsorbents. This paper presents a critical review of the development of the preparation of MMOFs with various structures, including core-shell structure, embedding structure, mixing structure and graphene structure. Particular emphasis is given to their application in environmental catalyst and adsorbents.
\end{abstract}

Keywords: magnetic, metal-organic framework, composites, environmental analysis, environmental remediation doi: $10.1360 / \mathrm{N} 032016-00199$ 